\title{
Advancing Dose-Response Assessment Methods for Environmental Regulatory Impact Analysis: A Bayesian Belief Network Approach Applied to Inorganic Arsenic
}

Joseph W. Zabinski, Gonzalo Garcia-Vargas, Marisela Rubio-Andrade, Rebecca C. Fry, and Jacqueline MacDonald Gibson*

\begin{abstract}
Dose-response functions used in regulatory risk assessment are based on studies of whole organisms and fail to incorporate genetic and metabolomic data. Bayesian belief networks (BBNs) could provide a powerful framework for incorporating such data, but no prior research has examined this possibility. To address this gap, we develop a BBN-based model predicting birthweight at gestational age from arsenic exposure via drinking water and maternal metabolic indicators using a cohort of 200 pregnant women from an arsenic-endemic region of Mexico. We compare BBN predictions to those of prevailing slope-factor and reference-dose approaches. The BBN outperforms prevailing approaches in balancing false-positive and false-negative rates. Whereas the slope-factor approach had 2\% sensitivity and $99 \%$ specificity and the reference-dose approach had $100 \%$ sensitivity and $0 \%$ specificity, the BBN's sensitivity and specificity were $71 \%$ and 30\%, respectively. BBNs offer a promising opportunity to advance health risk assessment by incorporating modern genetic and metabolomic data.
\end{abstract}

\section{INTRODUCTION}

Every major new US environmental regulation must undergo cost-benefit analysis to establish whether anticipated public health and environmental gains outweigh regulatory costs. ${ }^{1}$ If costs outweigh benefits, the Office of Management and Budget may return the proposed regulation to the Environmental Protection Agency (EPA) for modification or withdrawal. ${ }^{2,3}$ In order to predict health benefits, cost-benefit analysts rely on doseresponse functions. These functions predict the number of deaths and illnesses in a population exposed to contaminants. If dose-response functions are inaccurate, the resulting benefits estimates could be either too high, leading to inefficient regulations, or too low, leading to regulations insufficient to protect public health.

For most contaminants, dose-response functions used for regulatory impact analyses are based on decades-old data collected in studies of laboratory rodents or, in a few cases, human populations. ${ }^{4,5}$ Dose-response functions for carcinogens assume a linear relationship

\footnotetext{
* The corresponding author can be reached at jackie.macdonald@unc.edu, or at (919) 966-7892. SUPPORTING INFORMATION

Supporting Information Available: description of sensitivity analysis metrics used; relative influence of network parameters on target node; summary statistics of the nodes and variables of the BBN and the regression model
} 
between contaminant exposure and the lifetime probability of cancer. That is, to predict cancer risks, analysts multiply the estimated exposure dose by a constant known as the "cancer slope factor." For all regulations other than those involving ambient air quality, dose-response assessments for non-carcinogenic effects are categorical: if the exposure dose is above a threshold known as the reference dose (RfD), then the exposed individual is assumed to be at risk, while exposures below the RfD are assumed to pose zero risk. These prevailing dose-response functions fail to incorporate modern biomedical data that have arisen from new analytical technologies, such as methods for sequencing DNA, analyzing DNA expression, and characterizing metabolic profiles. In addition, the approaches used for cancer and non-cancer health outcomes are inconsistent (the latter assuming a categorical response with a threshold and the former assuming a linear, no-threshold response). Due to these and other concerns, Congress has held hearings on and called for National Research Council reviews of EPA's processes for developing dose-response functions, ${ }^{6}$ heightening the urgency of developing alternatives that can incorporate complex biomedical data while employing a consistent process for cancer and non-cancer risks.

We propose that Bayesian belief networks (BBNs) could provide a platform for developing dose-response functions that incorporate modern biomedical data. BBNs emerged from the artificial intelligence field in the 1980 s as a means to support causal inference. ${ }^{7}$ Although ecologists have long used BBNs in resource management and risk assessment, ${ }^{8-11}$ to our knowledge BBNs have not been previously used in human health risk assessment for environmental regulatory applications. We demonstrate the development of a BBN-based dose-response model for analyzing the risk of lower birthweight for gestational age as a function of arsenic exposure via drinking water, metabolic data, and demographic factors. We parameterize and test our model using data from a cohort of 200 mothers and newborns in an arsenic-endemic region of Mexico. We compare the BBN's predictive capability to that of prevailing dose-response assessment methods.

\section{MATERIALS AND METHODS}

\section{Maternal Birth Cohort}

To compare a BBN-based dose-response assessment approach to the prevailing RfD and slope factor approaches, we used maternal health, demographic, environmental exposure, and birth outcome data from the Biomarkers of Exposure to Arsenic (BEAR) prospective pregnancy cohort. ${ }^{12}$ This cohort was recruited in 2011-2012 from Gomez Palacio, Mexico, where 400,000 people are exposed to high arsenic levels. ${ }^{13}$ Participant recruitment methods are described elsewhere. ${ }^{12}$

For each participant, social workers collected information on age, education, smoking and alcohol consumption behaviors during pregnancy, seafood consumption, and sources of drinking and cooking water. Attending physicians reported infant birthweights and gestational ages at delivery. Maternal urine samples collected at delivery were analyzed for total, inorganic, and methylated arsenic as described in Laine et al. (2015). ${ }^{14}$ 


\section{Birth Outcome Measure}

Infants with birthweights below the $10^{\text {th }}$ percentile for gestational age are typically classified as small for gestational age. ${ }^{15}$ We calculated the small-for-gestational age cutoff values using a World Health Organization tool. ${ }^{16}$ Using this definition, only 14 infants in the cohort were small for gestational age. Due to the small sample size, we developed dose-response models to predict the probability that the birthweight-to-gestational-age (BWGA) ratio was below the $25^{\text {th }}$ percentile, an outcome that we designate as "lower BWGA." Of the 200 infants, 57 were designated as lower BWGA.

\section{Reference Dose Approach}

Current US environmental policies define the RfD for assessing noncancer risks as ${ }^{17}$

$$
R f D=\frac{N O A E L}{U F_{\text {inter }} * U F_{\text {intra }} * U F_{\text {other }}}
$$

where NOAEL is the no observable adverse effects level (the largest dose at which no statistically significant effects are observed) and the $U F$ s are uncertainty factors accounting for interspecies extrapolation, intra-species differences, and uncertainty sources. The current arsenic RfD, $0.3 \mu \mathrm{g}$ arsenic/(kg body weight-day), was derived from 1968 data on hyperpigmentation and keratosis incidence in a Taiwanese population exposed to arsenic in drinking water. ${ }^{18}$ Because this RfD does not consider birth outcomes, we computed an RfD for the BEAR cohort using Equation 1. Consistent with the current RfD, we assumed $U F_{\text {inter }}=U F_{\text {intra }}=1$ and $U F_{\text {other }}=3 .{ }^{19}$ In addition, we compared the BBN-based approach with the current regulatory RfD. For both analyses, we assumed that pregnant women drink 0.872 liters/day and weigh $75 \mathrm{~kg} .{ }^{20}$ All women exposed at levels above the RfD were assumed to be at risk of delivering an infant with lower BWGA. Sensitivity and specificity were estimated by comparing the resulting assignment of risk status to the true birth outcome for each participant.

\section{Slope Factor Approach}

The current arsenic slope factor, 1,500 kg-day/ $\mu \mathrm{g}$, was developed from data collected in 1968 and 1977 on skin cancer prevalence as a function of arsenic exposure in the previously mentioned arsenic-endemic region of Taiwan. Because this slope factor is not applicable for estimating adverse birth outcomes, we estimated a slope factor for lower BWGA risk using the BEAR cohort. Consistent with the general approach for estimating cancer risk slope factors, we computed a maximum likelihood estimator (using Stata) by regressing BWGA against inorganic arsenic exposure concentration in drinking water along with other covariates (total maternal urinary arsenicals, maternal urinary monomethylated arsenic, age, education, alcohol and seafood consumption during pregnancy, smoking during pregnancy, and infant gender). Covariates were chosen based on a prior BEAR cohort analysis. ${ }^{12}$ BWGA and all covariates measured on continuous scales were treated as continuous. We tested sensitivity and specificity using via leave-one-out cross-validation: each cohort member was removed from the data set, a regression model was fitted to the remaining 199 members, the model was used to predict BWGA for the corresponding test subject, and this 
estimate was converted to an indicator of lower BWGA status and compared against the case's true status.

\section{BBN Approach}

A BBN that predicts lower BWGA from the same covariates used in the regression model for the slope factor analysis was constructed using BayesiaLab (Laval, France) software. To the explanatory variables in the regression model, we added urinary inorganic and dimethylated arsenic, which were excluded from the regression model due to multicollinearity. In brief, a BBN is a probabilistic model represented as a directed acyclic graph in which nodes are variables and edges represent causal dependencies. ${ }^{7,21}$ A fully parameterized BBN represents the joint probability distributions among the variables. Our team's biomedical experts developed the BBN structure based on known or suspected mechanisms through which ingested inorganic arsenic is converted to potentially hazardous arsenic metabolites. The BEAR cohort data were then used to parameterize the model. All BBN variables were discretized (Table S2). We calibrated the posterior probability threshold above which lower BWGA status is assigned to maximize sensitivity first and then specificity. Sensitivity and specificity were tested using a leave-one-out cross-validation approach, in which the BBN was fitted to 199 cases and its prediction of lower BWGA status in the remaining case was compared to the case's actual status.

\section{RESULTS AND DISCUSSION}

\section{Fitted Models}

To estimate an RfD relating lower BWGA risk to arsenic exposure, we divided the LOAEL in the BEAR cohort $(0.461 \mu \mathrm{g} / \mathrm{liter}$, the detection limit) by an uncertainty factor of three, resulting in an RfD of $0.00179 \mu \mathrm{g} / \mathrm{kd}$-day. This estimated RfD is more than two orders of magnitude less than the current US regulatory RfD of $0.3 \mu \mathrm{g} / \mathrm{kg}$-day, which is based on skin hyperpigmentation and keratosis.

To estimate a model consistent with the slope factor approach, we used a multivariate linear regression to predict BWGA from the inorganic arsenic concentration in drinking water and other covariates summarized in Table S1. Consistent with prior research on this cohort, ${ }^{12}$ the urinary concentration of monomethylated arsenic was highly significant $(\mathrm{p}=0.003)$, and the drinking water arsenic concentration was marginally significant $(\mathrm{p}=0.107)$ (Table $\mathrm{S} 1)$.

We fitted a BBN model to predict lower BWGA status as a function of the same variables used in the regression model plus two additional descriptors of maternal arsenic metabolism (inorganic and dimethylated arsenic concentrations in urine) that could not be included in the regression model due to multicollinearity (Figure 1). A sensitivity analysis showed mother's age, infant gender, and urinary concentration of monometheylated arsenic have the greatest information value for predicting BWGA status (Figure S1).

\section{Model Sensitivity and Specificity}

The RfD derived from the BEAR cohort predicted that every infant was in the lower BWGA category. Sensitivity and specificity were therefore $100 \%$ and $0 \%$ (Figure 2). In contrast, 
using EPA's current RfD (28.6 $\mu \mathrm{g} / \mathrm{L})$, sensitivity and specificity were $25 \%$, and $73 \%$, respectively (Figure 2). Therefore, the current RfD misclassified $75 \%$ of lower BWGA cases.

The slope factor approach also yielded skewed results. Though the data set contained 57 lower BWGA cases, the regression predicted three such cases (and of these three, only one was an actual case). The corresponding sensitivity and specificity were $2 \%$ and $99 \%$, respectively (Figure 2).

In contrast to the other methods, the BBN achieved a more even balance between sensitivity (71\%) and specificity (30\%; Figure 2).

As a sensitivity analysis of model performance, cross-validation was repeated with $10 \%$ of the data used as a testing set. The BBN model still outperformed the other methods in balancing sensitivity and specificity. The sensitivities of the RfD, regression, and BBN models were $100 \%, 2 \%$, and $65 \%$, respectively; specificities were $0 \%, 98 \%$, and $29 \%$.

\section{Relevance}

Accurate risk assessment requires methods that balance the public health costs of false negatives with the potential excess regulatory costs of false positives. As demonstrated, our BBN model outperformed the RfD and slope factor methods in balancing sensitivity and specificity when predicting lower birthweight risk as a function of inorganic arsenic exposure in water. Specifically, the BBN achieved higher sensitivity (71\%) than the slope factor approach (2\%) and higher specificity (30\%) than the RfD approach (0\%).

Furthermore, unlike the RfD approach, the BBN also was able to incorporate data on maternal arsenic metabolism, thought to be an important factor in fetal risk. ${ }^{12}$ Unlike the slope factor model, the BBN included multiple, correlated measures of maternal metabolism.

We hypothesize that the BBN outperforms the conventional methods for several reasons. The binary RfD approach cannot account for covariates or metabolic factors affecting infant risk. While the regression model includes metabolic indicators and demographic variables, its ability to do so is limited by the requirement of independence among predictors, the assumed linear relationship between BWGA and predictors, and limited ability to detect complex interactions. ${ }^{22}$ The regression model's low $\mathrm{R}^{2}(0.122)$ indicates its limited predictive power. The BBN, in contrast, is distribution-free and can account for linear and nonlinear relationships (or even relationships that may have both linear and nonlinear regions) along with correlations and complex interactions among predictor variables.

Although this work represents the first comparison of BBN-based and prevailing methods for human health dose-response assessment in an environmental context, a number of previous studies have compared BBN performance to that of prevailing predictive methods. For example, multiple studies have explored the capability of BBNs to predict health outcomes under alternative medical treatment regimes. ${ }^{23-28}$ Similar to in our study, many of these studies found that BBNs outperformed conventional prognostic methods. As an example, Forsberg et al. (2011) demonstrated that a BBN outperformed conventional 
approaches in estimating survival in patients with operable skeletal metastases. ${ }^{28}$ In addition, other studies of medical outcomes have compared the performance of BBNs to that of regression models and found BBN performance comparable to or better than that of regression approaches. As an example, Stojadinovic et al. found that a BBN for estimating healthcare outcomes in severely wounded veterans was comparable in predictive capability to a logistic regression model. ${ }^{25}$ Despite their comparable performance, the authors recommended use of the BBN over regression because it was able to reveal associations between factors that were not evident in the regression and because its intuitive graphical structure could help clinicians understand causes of alternative health outcomes. In addition to medical applications, applications in ecological risk assessment have demonstrated superior performance of BBNs in comparison to traditional methods. As an example, Walton and Meidinger (2006) found that a BBN method for classifying ecosystem types in mapping applications outperformed the prevailing approach, which was based on expert review of various ecosystem measures. ${ }^{29}$ This evidence suggests that BBNs deserve further consideration for dose-response modeling, due to both their intuitive structure and their powerful analytic capabilities.

The major limitation of this study is the small size of the data set. In addition, the newborns in this cohort were generally of healthy weight, perhaps due to the "Mexican paradox," the tendency for Mexican newborns to be at lower risk for underweight birth than expected from demographic data. ${ }^{30}$ Nonetheless, our model was more effective than both prevailing methods in classifying cases according to lower BWGA status. Future research with larger cohorts and/or additional variables representing the mechanisms through which arsenic acts on BWGA should improve model performance.

We have demonstrated that a BBN model outperforms prevailing RfD and regression-based slope factor approaches in predicting birthweight outcomes from arsenic exposure and maternal metabolic data. The BBN achieves this superior performance by incorporating information in a nonlinear, nonparametric structure that offers greater freedom than traditional approaches. In addition, the organization of the BBN is visually intuitive: relationships between variables are mapped clearly, and the structure lends itself to the development of risk assessment tools that may be more user-friendly than those currently available. Unlike the separate RfD and slope factor approaches, the BBN model also offers a unified and consistent way of assessing both cancer and non-cancer risks. Perhaps most importantly, BBNs allow for the incorporation of modern biomedical data into doseresponse functions, offering a promising opportunity for advancing dose-response assessment and health environmental regulatory impact analysis.

\section{Supplementary Material}

Refer to Web version on PubMed Central for supplementary material.

\section{REFERENCES}

1. Executive Office of the President. Executive Order 12866 of September 30, 1993: Regulatory Planning and Review. 1993 
2. General Accounting Office. OMB's Role in Reviews of Agencies' Draft Rules and the Transparency of Those Reviews. 2003

3. Ackerman F, Heinzerling L. Pricing the Priceless: Cost-Benefit Analysis of Environmental Protection. Univ. PA. Law Rev. 2002; 150(5):1553.

4. Abt E, Rodricks JV, Levy JI, Zeise L, Burke T. a. Science and Decisions: Advancing Risk Assessment. Risk Anal. 2010; 30(7):1028-1036. [PubMed: 20497395]

5. U.S. National Research Council. Science and Decisions: Advancing Risk Assessment. National Academy Press; Washington, D.C.: 2009.

6. National Research Council, US. Reveiw of EPA's Integrated Risk Information System (IRIS) Process. National Academies Press; Washington, D.C.: 2014.

7. Pearl, J. Probabilistic Reasoning in Intelligent Systems: Networks of Plausible Inference. Morgan Kaufmann Publishers, Inc.; San Francisco, Calif.: 1988.

8. Pollino CA, Woodberry O, Nicholson A, Korb K, Hart BT. Parameterisation and Evaluation of a Bayesian Network for Use in an Ecological Risk Assessment. Environ. Model. Softw. 2007; 22(8): 1140-1152.

9. Bayliss P, van Dam RA, Bartolo RE. Quantitative Ecological Risk Assessment of the Magela Creek Floodplain in Kakadu National Park, Australia: Comparing Point Source Risks from the Ranger Uranium Mine to Diffuse Landscape-scale Risks. Hum Ecol Risk Assess. 2012; 18(1):115-151.

10. Ayre KK. A Bayesian Approach to Landscape Ecological Risk Assessment Applied to the Upper Grande Ronde Watershed, Oregon. Hum Ecol Risk Assess. 2012; 18(5):946-970.

11. McCann RK, Marcot BG, Ellis R. Bayesian Belief Networks: Applications in Ecology and Natural Resource Management. Can. J. For. Res. 2006; 36(12):3053-3062.

12. Laine JE, Bailey KA, Rubio-Andrade M, Olshan AF, Smeester L, Drobna Z, Herring AH, Styblo M, Garcia-Vargas GG, Fry RC. Maternal Arsenic Exposure, Arsenic Methylation Efficiency, and Birth outcomes in the biomarkers of Exposure to ARsenic (BEAR) Pregnancy Cohort in Mexico. Environ. Health Perspect. 2015; 123(2):186-192. [PubMed: 25325819]

13. Armienta MA, Segovia N. Arsenic and Fluoride in the Groundwater of Mexico. Environ. Geochem. Health. 2008; 30(4):345-353. [PubMed: 18335171]

14. Rager JE, Bailey KA, Smeester L, Miller SK, Parker JS, Laine JE, Drobna Z, Currier J, Douillet C, Olshan A, et al. Prenatal Arsenic Exposure and the Epigenome: Altered microRNAs Associated with Innate and Adaptive Immune Signaling in Newborn Cord Blood. Environ. Mol. Mutagen. 2014; 55:196-208. [PubMed: 24327377]

15. Kendig, JW.; Nawab, U. Small-for-Gestational-Age (SGA). Merck \& Co.; Kenilworth, NJ: 2015.

16. World Health Organization. Weight Percentiles Calculator.

17. US Environmental Protection Agency. Reference Dose (RfD): Description and Use in Health Risk Assessments. Washington, D.C.: 1993.

18. U.S. Environmental Protection Agency National Center for Environmental Assessment. Integrated Risk Information System (IRIS).

19. USEPA. Chemical Assessment Summary: Arsenic (Inorganic).

20. U.S. Environmental Protection Agency. Exposure Factors Handbook: 2011 Edition. Washington, D.C.: 2011.

21. Lauritzen SL, Spiegelhalter D. Local Computations with Probabilities on Graphical Structures and Their Application to Expert Systems. J. R. Stat. Soc. 2010; 50(2):157-224.

22. Su C, Andrew A, Karagas MR, Borsuk ME. Using Bayesian Networks to Discover Relations between Genes, Environment, and Disease. BioData Min. 2013; 6(1):6. [PubMed: 23514120]

23. Wang X-H, Zheng B, Good WF, King JL, Chang Y-H. Computer-assisted Diagnosis of Breast Cancer Using a Data-driven Bayesian Belief Network. Int. J. Med. Inform. 1999; 54(2):115-126. [PubMed: 10219951]

24. Zheng B, Ramalingam P, Hariharan H, Leader JK, Gur D. Prediction of Near-Term Breast Cancer Risk using a Bayesian Belief Network. 2013; 8673:86731F.

25. Stojadinovic A, Eberhardt J, Brown TS, Hawksworth JS, Gage F, Tadaki DK, Forsberg JA, Davis TA, Potter BK, Dunne JR, et al. Development of a Bayesian Model to Estimate Health Care 
Outcomes in the Severely Wounded. J. Multidiscip. Healthc. 2010; 3:125-135. [PubMed: 21197361]

26. Stojadinovic A, Nissan A, Eberhardt J, Chua TC, Pelz JOW, Esquivel J. Development of a Bayesian Belief Network Model for Personalized Prognostic Risk Assessment in Colon Carcinomatosis. Am. Surg. 2011; 77(2):221-230. [PubMed: 21337884]

27. Liao Y, Wang J, Guo Y, Zheng X. Risk Assessment of Human Neural Tube Defects Using a Bayesian Belief Network. Stoch. Environ. Res. Risk Assess. 2010; 24(1):93-100.

28. Forsberg JA, Eberhardt J, Boland PJ, Wedin R, Healey JH. Estimating Survival in Patients with Operable Skeletal Metastases: An Application of a Bayesian Belief Network. PLoS One. 2011; 6(5):1-7.

29. Walton A, Meidinger D. Capturing Expert Knowledge for Ecosystem Mapping using Bayesian Networks. Can. J. For. Res. 2006; 36(12):3087-3103.

30. Buekens P, Notzon F, Kotelchuck M, Wilcox A. Why do Mexican Americans Give Birth to Few Low-birth-weight Infants? Am. J. Epidemiol. 2000; 152(4):347-351. [PubMed: 10968379] 


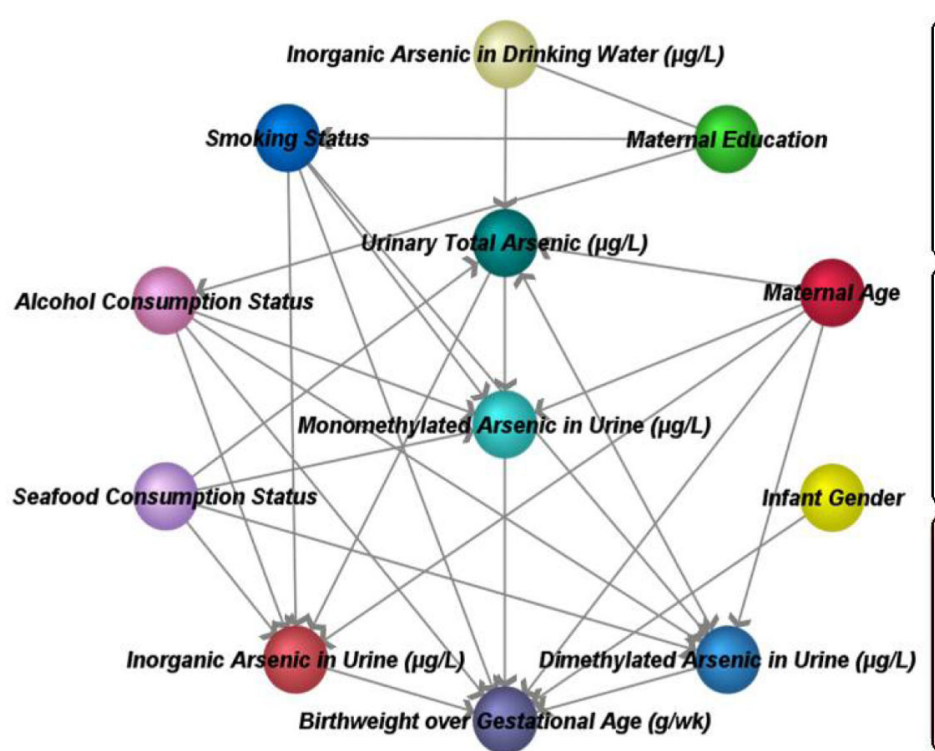

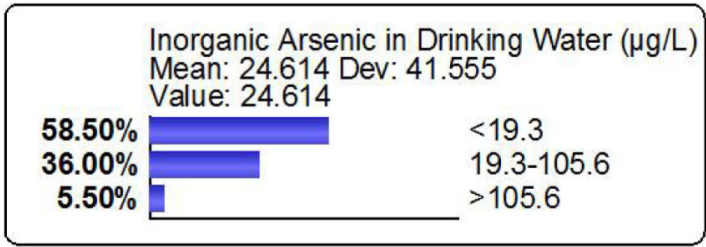

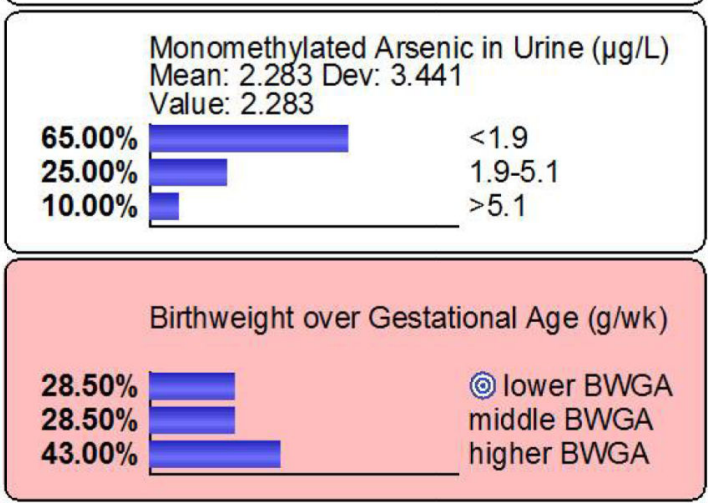

Figure 1.

Bayesian belief network model for predicting the risk of lower birthweight for gestational age as a function of maternal arsenic exposure, arsenic metabolism, and behavioral and demographic factors. The lowest node predicts birthweight divided by gestational age (BWGA) as a function of all of the other variables in the network; the target symbol indicates that the lower BWGA node state is the outcome of interest. The corresponding belief bars show probabilities of lower BWGA $\left(<25^{\text {th }}\right.$ percentile $)$, middle BWGA $\left(25^{\text {th }}-75^{\text {th }}\right.$ percentile), or higher BWGA $\left(>75^{\text {th }}\right.$ percentile) conditional on baseline states of the other nodes. Underlying all nodes are conditional probability tables fitted to the data set used in this study. Updated predictions of BWGA can be obtained by specifying the state of any node or set of nodes, and by performing the necessary probability calculus. While a number of nodes influence BWGA directly, several others' effects are mediated by intermediates. The choice of structure was made to elicit predictive power while also maintaining biological plausibility. 
- Sensitivity (True Positive Rate)

$\mathbf{\Delta}$ Specificity (True Negative Rate)

RfD approach (EPA value)

$\mathrm{RfD}$ approach (calculated)

Slope factor approach

BBN
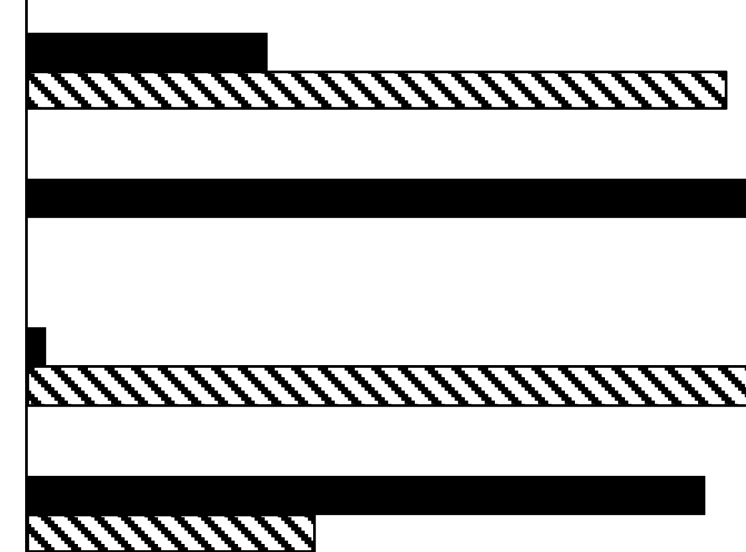

N1N1ND

$\begin{array}{llllll}0 & 20 \% & 40 \% & 60 \% & 80 \% & 100 \%\end{array}$

Figure 2.

Sensitivity and specificity of alternative dose-response functions for predicting lower birthweight for gestational age. $\mathrm{RfD}=$ reference dose; $\mathrm{BBN}=$ Bayesian belief network. 